\section{Investigación original}

dx.doi.org/10.35366/CMA201C

\title{
Dexmedetomidina subcutánea. ¿Es útil en el perioperatorio del paciente pediátrico?
}

\author{
Subcutaneous dexmedetomidine. Is it useful in the \\ perioperative of the pediatric patient?
}

\author{
Dra. Ana Elsa Álvarez-Betancourt, ${ }^{*}$ Dr. Eloy Sánchez-Hernández, ${ }^{\ddagger}$ \\ Dra. Brenda Guadalupe López-González, ${ }^{\S}$ Dr. Óscar Armando Rodríguez-Moreno"
}

RESUMEN. Introducción: La dexmedetomidina es un alfa 2 agonista, al cual se le han atribuido diversos efectos clínicos (antinociceptivos, hipnótico-sedantes, anestésicos, cardiovasculares, ventilatorios, renales, endocrinos y oculares), variados mecanismos de acción, y versatilidad en sus vías de administración, por lo que ha sido utilizada como adyuvante durante el período perioperatorio, ya que proporciona sedación, ansiólisis, prevención de delirio y analgesia. En este estudio, se evaluó exclusivamente la administración subcutánea como adyuvante, sus implicaciones sobre el dolor y la comodidad en el postoperatorio. Material y métodos: Se realizó una serie de casos de forma prospectiva, observacional, descriptiva, en donde se estudiaron pacientes de 3-15 años de edad, de ambos géneros, programados para cirugía de abdomen bajo, ASA I-II. Se dividieron en cuatro grupos: grupo I recibió placebo; II, III y IV clorhidrato de dexmedetomidina de $1,1.5$ y $2 \mu \mathrm{g} / \mathrm{kg}$, respectivamente. El placebo o clorhidrato de dexmedetomidina se administraron vía subcutánea en región deltoidea. En el postoperatorio inmediato se tomaron en cuenta, por única vez, la escala de sedación, delirio y confort, para valorar durante la emersión el impacto de la dosis de dexmedetomidina. Después se realizó la medición y registro en tres tiempos distintos de los parámetros hemodinámicos ( $\mathrm{TA}, \mathrm{FC}, \mathrm{SpO}_{2}$ ), así como la intensidad del dolor; la primera al finalizar el procedimiento quirúrgico, a las 12 y a las 24 horas. Conclusiones: La administración subcutánea de dexmedetomidina en el paciente pediátrico es una alternativa segura y eficaz, que preserva la función respiratoria, con adecuado grado de sedación, y estabilidad hemodinámica, mejorando la analgesia (hasta 24 horas) posterior al evento quirúrgico, menor agitación y delirio en el postoperatorio, lo que resulta en mayor comodidad.

ABSTRACT. Introduction: Dexmedetomidine is an alpha 2 agonist, several clinical effects has been attributed (antinociceptive, hypnotic-sedative, anesthetic, cardiovascular, ventilatory, renal, endocrine and ocular), varied mechanisms of action, and versatility in its administration pathways. It has been used as adjuvant during the perioperative period providing sedation, anxiolysis, delirium prevention and analgesia. In this study we evaluated exclusively subcutaneous administration as an adjuvant, its implications on pain and postoperative comfort (PO). Material and methods: A prospective, observational, descriptive series of cases was performed. Patients 3-15 years of age, scheduled for low abdominal surgery, both genders, ASA I, II. They were divided into four groups: Group I. placebo, group II, III and IV received dexmedetomidine hydrochloride 1, 1.5 and $2 \mu \mathrm{g} / \mathrm{kg}$ respectively. The placebo or dexmedetomidine hydrochloride was administered subcutaneously in the deltoid region. The sedation, delirium and comfort scale were titrated in the immediate postoperative $P O$ to assess the impact of the dose of dexmedetomidine during the emersion. Subsequently, three evaluations of the hemodynamic parameters ( $\left.\mathrm{TA}, \mathrm{FC}, \mathrm{SpO}_{2}\right)$ were performed, as well as the intensity of the pain, the first one at the end of the surgical procedure, 12 and at 24 hours later. Conclusions: Subcutaneous administration of dexmedetomidine in the pediatric patient is a safe and effective alternative, preserving respiratory function, with adequate sedation, hemodynamic stability, improving analgesia (up to 24 hours) after the surgical event, less agitation and delirium in the postoperative resulting in greater comfort.

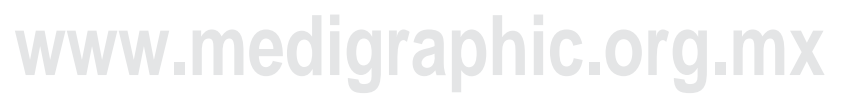

Anestesiología

Enero-Marzo 2020

Vol. 43. No. 1. pp 16-22

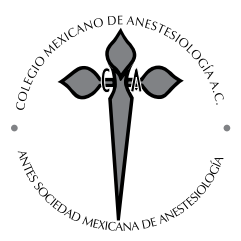

* Residente de segundo año de Anestesiología Pediátrica. Hospital Civil de Guadalajara «Fray Antonio Alcalde».

‡ Anestesiólogo Pediatra, adscrito al Servicio de Anestesiología, Profesor Titular del Curso de Anestesiología Pediátrica. Hospital Civil de Guadalajara «Fray Antonio Alcalde».

$\S$ Anestesiólogo Pediatra, adscrito al servicio de Anestesiología Pediátrica. Unidad Médica de Alta Especialidad, Centro Médico Nacional de Occidente. Hospital de Pediatría, Guadalajara Jalisco. México. " Anestesiólogo Adscrito al servicio de Anestesiología. Unidad Médica de Alta Especialidad, Centro Médico Nacional de Occidente, Guadalajara Jalisco. México.

\section{Palabras clave:}

Dexmedetomidina, analgesia, subcutánea, sedación,dolor, pediatría

\section{Keywords:}

Dexmedetomidine, analgesia, subcutaneous, sedation, pain, pediatrics

\section{Solicitud de Sobretiros:}

Dr. Eloy Sánchez-Hernández Hospital Civil de Guadalajara

"Fray Antonio Alcalde"

Calle Hospital Número 278,

Colonia El Retiro, Guadalajara, Jalisco, México.

Tel. +52 39424400, ext 41045

E-mail: dreloy124@hotmail.com

Recibido para publicación: 08-01-2019

Aceptado para publicación:

09-06-2019 


\section{INTRODUCCIÓN}

I a finalidad de nuestra práctica como anestesiólogos es brindar a nuestros pacientes un evento anestésico confortable, seguro (manteniendo su estabilidad hemodinámica en el transoperatorio), junto con un adecuado manejo y control del dolor postoperatorio (PO). Por lo que nos auxiliamos de la administración de fármacos adyuvantes a los anestésicos, para lograr una correcta medicación preanestésica y ansiólisis, estabilidad hemodinámica y analgesia ${ }^{(1-5)}$. La dexmedetomidina es un fármaco que ha sido utilizado con este propósito, y puede emplearse con seguridad en el paciente pediátrico.

Es un derivado imidazólico, alfa 2 adrenérgico, con afinidad sobre el receptor alfa 1: alfa 2 de (1:1600), lipofílico, con propiedades sedantes, hipnóticas, analgésicas, que disminuye los requerimientos anestésicos y la liberación de catecolaminas ${ }^{(5-9)}$. Puede reducir la respuesta inflamatoria y la hiperalgesia resultado de la inflamación ${ }^{(10)}$.

Fue aprobada en 1999 por la FDA (Food and Drug Administration) para uso en Unidad de Cuidados Intensivos; posteriormente, se le atribuyeron propiedades analgésicas y como un agente para sedación cooperadora ${ }^{(6)}$. Esta condición es de interés para su uso dentro de la anestesia. Existen múltiples estudios clínicos donde se han empleado diversas vías de administración, atribuyéndosele muchas acciones en los diversos contextos en que ha sido utilizada; además de mecanismos de acción, efectos antinociceptivos, hipnóticosedantes, anestésicos, cardiovasculares, ventilatorios, renales, endocrinos y oculares ${ }^{(5)}$. Respecto a la población pediátrica, ha sido utilizada exitosamente en radiodiagnóstico, hemodinamia y manejo de vía aérea ${ }^{(1-3)}$. También se ha indicado para sedación en la Unidad de Cuidados Postanestésicos, para reducir la incidencia de agitación en niños manejados con sevoflurano. En la sedación quirúrgica, se han realizado craneotomías en pacientes pediátricos con el paciente despierto usando la dexmedetomidina ${ }^{(4,5)}$.

La aplicación de los medicamentos de forma parenteral son: intravenosa (IV), subcutánea (SC) e intramuscular, principalmente. En la vía subcutánea, la absorción ocurre por difusión sencilla, al seguir el gradiente que media entre el depósito del medicamento y el plasma. La velocidad depende del área de las membranas capilares, las cuales absorben el producto, y de la solubilidad de la sustancia en el líquido intersticial. Los canales acuosos relativamente grandes de la membrana endotelial permiten una difusión indiscriminada de moléculas, independiente de su liposolubilidad. Las moléculas grandes, como las de las proteínas, penetran con lentitud en la circulación a través de los conductos linfáticos. Después de una inyección subcutánea, la velocidad de absorción del fármaco suele ser constante y lenta, con lo cual proporciona un efecto sostenido.

La absorción transdérmica de un fármaco depende de la superficie sobre la que se aplica (integridad de piel) y de su liposolubilidad, ya que la epidermis se comporta como lipobarrera $^{(11)}$. La vía transdérmica ofrece ciertas ventajas sobre otras vías de administración. Un fármaco, al ser administrado por esta vía, tiene una liberación de forma sostenida y una absorción lenta, y alcanza una concentración plasmática estable por un período largo; por esta causa se han desarrollado formas transdérmicas de clonidina, fentanyl, nicotina y nitroglicerina ${ }^{(12)}$.

Existen pocas publicaciones sobre el uso subcutáneo e intradérmico de la dexmedetomidina; cuando ésta atraviesa la piel, se distribuye para traspasar las barreras celulares, mediante diferentes mecanismos como la filtración, difusión pasiva y difusión facilitada. Haciendo referencia a lo descrito previamente, podemos inferir que, por sus características liposolubles, tiene una adecuada absorción y propagación por esta vía ${ }^{(13)}$. Se ha observado una rápida absorción con amplia distribución tisular, cuando se han empleado dosis mediante vía subcutánea e intramuscular ${ }^{(5,6,12)}$.

Se ha determinado que posee un modelo farmacocinético bicompartimental de acuerdo con sus características; tiene una tasa de unión a las proteínas plasmáticas de 94\%, posee un metabolismo principalmente hepático, y es eliminada por vía renal en $95 \%(5,6)$.

\section{MATERIAL Y MÉTODOS}

Se realizó una serie de casos prospectiva, observacional y descriptiva. Se estudiaron 52 pacientes de entre 3-15 años de edad, programados para cirugía de abdomen bajo. Éstos fueron asignados mediante sistema aleatorio simple en forma secuencial a cada uno de los cuatro grupos (13 pacientes cada uno). Grupo I control, II Dex $1 \mu \mathrm{g} / \mathrm{kg}$, III Dex $1.5 \mu \mathrm{g} / \mathrm{kg}$ y IV Dex $2 \mu \mathrm{g} / \mathrm{kg}$.

La dexmedetomidina es utilizada cotidianamente en el paciente pediátrico como adyuvante anestésico, por lo que no se adiciona ningún riesgo al procedimiento.

Dado que no existen más que dos antecedentes en la literatura en los que se hace referencia a la vía subcutánea, y no hay evidencia escrita de la misma por la FDA, se consideró importante presentarlo para que fuera evaluado y avalado por el Comité de Investigación y Ética del Hospital Civil «Fray Antonio Alcalde» de Guadalajara, donde se valoraron los aspectos metodológicos y éticos del proyecto. Así como también se obtuvo el consentimiento informado por parte de los padres/tutores y se entregó el documento de confidencialidad.

Selección y descripción. Se incluyeron pacientes de 3 a 15 años, de ambos géneros, programados para cirugía de abdomen bajo, ASA I-II. No se aceptó a quienes estuvieran previamente orointubados, los que presentaron alguna complicación anestésica o quirúrgica, o que ameriten intubación orotraqueal postoperatoria y empleo de otros fármacos fuera de lo 
normal. Se excluyeron aquéllos con alergia al medicamento, los que presentaron reacción adversa al mismo y pacientes con infección en el sitio de la aplicación del medicamento.

Información técnica. Posterior a la valoración preanestésica y plan educacional por parte del anestesiólogo al paciente y padres/tutores, así como obtenida la firma del consentimiento informado, fueron asignados de forma aleatorizada simple en forma secuencial a uno de los cuatro grupos.

Grupo I. Placebo solución salina 0.9\%

Grupo II. Clorhidrato de dexmedetomidina $1 \mu \mathrm{g} / \mathrm{kg} \mathrm{SC}$

Grupo III. Clorhidrato dexmedetomidina $1.5 \mu \mathrm{g} / \mathrm{kg} \mathrm{SC}$

Grupo IV. Clorhidrato de dexmedetomidina $2 \mu \mathrm{g} / \mathrm{kg} \mathrm{SC}$

A su ingreso, fueron premedicados con midazolam 50 $\mu \mathrm{g} / \mathrm{kg} \mathrm{VO/IV}$. En quirófano, se monitorizaron de forma no invasiva (monitoreo tipo I). Después, se realizó la inducción de forma inhalatoria en los que no tenían acceso intravenoso, con sevoflurano a concentraciones variables más oxígeno al $100 \%$, para canalizar acceso venoso. Una vez conseguido, se complementó la inducción con fentanyl $3 \mu \mathrm{g} / \mathrm{kg}$ y lidocaína $1 \mathrm{mg} / \mathrm{kg}$, propofol $3 \mathrm{mg} / \mathrm{kg}$. El manejo de la vía aérea fue a través de intubación endotraqueal o con mascarilla laríngea ( $\mathrm{FiO}_{2} 50 \%$ ). El mantenimiento anestésico fue con sevoflurano/ propofol y fentanyl.

Posterior a la inducción anestésica, y previa asepsia y antisepsia de región deltoidea, se administró el placebo o clorhidrato de dexmedetomidina vía subcutánea con jeringa de insulina (1 mL de $27 \mathrm{G} \mathrm{x} 13 \mathrm{~mm}$ ), según el grupo asignado previamente. Durante el transanestésico se registraron los signos vitales de forma continua. Todos los sujetos de estudio recibieron dosis analgésica de paracetamol a $15 \mathrm{mg} / \mathrm{kg}$.

Se valoró por única vez en el postoperatorio inmediato la escala de sedación, delirio y confort, para evaluar durante la emersión el impacto de la dosis de dexmedetomidina. Luego se midieron y registraron, en tres tiempos distintos, los parámetros hemodinámicos (TAS, FC, $\mathrm{SpO}_{2}$ ), así como la intensidad del dolor; la primera al finalizar el procedimiento quirúrgico, a las 12 y a las 24 horas.

Estadística. Se realizó mediante análisis de medidas paramétricas cuantitativas, prueba $\mathrm{T}$ y análisis de varianza (ANOVA).

\begin{tabular}{lcccc}
\multicolumn{5}{c}{ Tabla 1: Distribución demográfica. } \\
& Grupo I & Grupo II & Grupo III & Grupo IV \\
\hline Edad (años) & $10 \pm 2$ & $8 \pm 4$ & $10 \pm 4$ & $8 \pm 5$ \\
Sexo & & & & \\
Masculino (n) & 10 & 9 & 11 & 5 \\
Femenino (n) & 3 & 4 & 2 & 8 \\
Peso (kg) & $42 \pm 14$ & $27 \pm 13$ & $41 \pm 21$ & $39 \pm 20$ \\
\hline
\end{tabular}

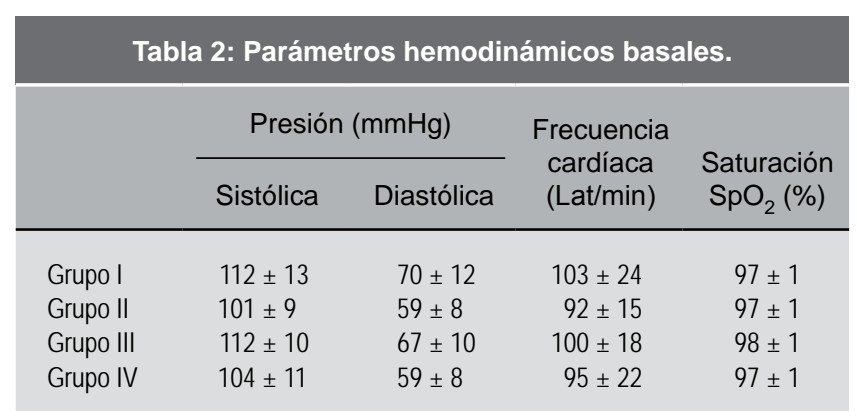

\begin{tabular}{|c|c|c|c|c|}
\hline & \multicolumn{2}{|c|}{ Presión (mmHg) } & \multirow{2}{*}{$\begin{array}{c}\text { Frecuencia } \\
\text { cardíaca } \\
\text { (Lat/min) }\end{array}$} & \multirow{2}{*}{$\begin{array}{c}\text { Saturación } \\
\mathrm{SpO}_{2}(\%)\end{array}$} \\
\hline & Sistólica & Diastólica & & \\
\hline Grupo I & $106 \pm 8$ & $68 \pm 13$ & $90 \pm 21$ & $97 \pm 1$ \\
\hline Grupo II & $97 \pm 8$ & $57 \pm 9$ & $88 \pm 20$ & $98 \pm 1$ \\
\hline Grupo III & $103 \pm 12$ & $60 \pm 11$ & $86 \pm 13$ & $98 \pm 1$ \\
\hline Grupo IV & $101 \pm 10$ & $58 \pm 8$ & $82 \pm 15$ & $97 \pm 1$ \\
\hline
\end{tabular}

\section{RESULTADOS}

Se incluyeron 52 pacientes, de entre 3 y 15 años, ambos sexos (Tabla 1).

Se registraron y compararon los parámetros hemodinámicos (tensión arterial, frecuencia cardíaca y saturación de oxígeno), tomando en cuenta sus parámetros basales al finalizar el procedimiento (Tabla 2), y se compararon con los resultados obtenidos a las 12 horas (Tabla 3) y 24 horas (Tabla 4), mostrándose los promedios obtenidos en cada grupo. Con base en los resultados obtenidos, no se encontró evidencia estadísticamente significativa entre cada uno de los grupos, y al contrastar a cada grupo con su registro basal, no se evidenciaron variaciones estadísticamente significativas entre ambos.

Se aplicó prueba $\mathrm{T}$ para análisis de variables, definiendo un valor crítico de $\mathrm{T}$ de 1.78, no se encontró diferencia estadísticamente significativa entre los grupos (Figura 1).

Para la valoración de la sedación, dolor, delirio postoperatorio y confort se emplearon las escalas de Ramsay, WongBaker, delirio del despertar postoperatorio y la de Confort, respectivamente (Figuras 2 a 4).

$\mathrm{Al}$ analizar los datos obtenidos en cada grupo, se puede observar que en el puntaje de la escala Ramsay hubo diferencia significativa, entre el grupo placebo y los tres grupos de dexmedetomidina. El análisis específico de los grupos de estudio (II, III y IV) no mostró diferencia estadísticamente significativa entre ellos, teniendo un comportamiento similar. 
Para los puntajes de la escala de delirium al despertar, fue evidente una disminución en el grupo II, respecto al resto de los grupos. Por su parte, los grupos III y IV que correspondieron a las dosis mayores de dexmedetomidina tuvieron un aumento en el puntaje de la escala de delirium, respecto al grupo I; sin embargo, dichas diferencias no probaron ser estadísticamente significativas.

Los resultados de la escala Confort fueron categorizados en tres rubros: profundo, óptimo e inadecuado. Se comparó la incidencia de cada una de las categorías en cada grupo (Tabla 5).

$\mathrm{Al}$ comparar los cuatro grupos se encontró diferencia estadísticamente significativa, con respecto al grupo I.

Referente al dolor postoperatorio, se evaluó la escala de caras de Wong-Baker a las 12 y 24 horas del postquirúrgico, y se encontró que, en la totalidad de los grupos, la intensidad del dolor no fue mayor a cuatro. Es importante mencionar

\begin{tabular}{|c|c|c|c|c|}
\hline & \multicolumn{2}{|c|}{ Presión (mmHg) } & \multirow{2}{*}{$\begin{array}{c}\text { Frecuencia } \\
\text { cardíaca } \\
\text { (Lat/min) }\end{array}$} & \multirow{2}{*}{$\begin{array}{l}\text { Saturación } \\
\mathrm{SpO}_{2}(\%)\end{array}$} \\
\hline & Sistólica & Diastólica & & \\
\hline Grupo I & $105 \pm 4$ & $71 \pm 13$ & $82 \pm 12$ & $97 \pm 1$ \\
\hline Grupo II & $102 \pm 8$ & $63 \pm 6$ & $92 \pm 13$ & $97 \pm 1$ \\
\hline Grupo III & $100 \pm 28$ & $66 \pm 11$ & $84 \pm 7$ & $98 \pm 1$ \\
\hline Grupo IV & $104 \pm 6$ & $64 \pm 7$ & $84 \pm 14$ & $97 \pm 1$ \\
\hline
\end{tabular}

que el manejo del dolor en el postoperatorio fue mediante paracetamol y/o metamizol, y únicamente un paciente requirió una dosis de rescate con morfina IV.

Al realizar la comparativa entre los grupos, se encontró diferencia estadísticamente significativa, con respecto al que recibió el placebo, y en todos los grupos de dexmedetomidina hubo una intensidad del dolor similar (puntaje 3), menor que en el grupo placebo (puntaje 4). A las 24 horas, los grupos I y II se mantuvieron sin cambios, en relación al registro previo (puntaje 4 y 3 , respectivamente), no teniendo significancia estadística, pero los grupos con dosis mayor de dexmedetomidina III y IV $(1.5$ y $2 \mu \mathrm{g} / \mathrm{kg})$ presentaron una disminución en el puntaje de 2 para los grupos III y IV (Figura 5).

Podemos ver la evolución y comportamiento hemodinámico de cada uno de los grupos respecto a sus parámetros hemodinámicos en la Figura 1.

\section{DISCUSIÓN}

Existen múltiples estudios que demuestran la efectividad de la dexmedetomidina aplicada en el perioperatorio con buenos resultados en general.

La dexmedetomidina es un alfa 2 agonista, al que se le han atribuido diversos efectos clínicos, el receptor alfa 2 adrenérgico media sus efectos a través de la activación de proteínas G, con lo que hay inhibición de adenilciclasa y reducción de la concentración de 3’5' AMPc. Se han sugerido otros mecanismos de acción alternativos, como la apertura

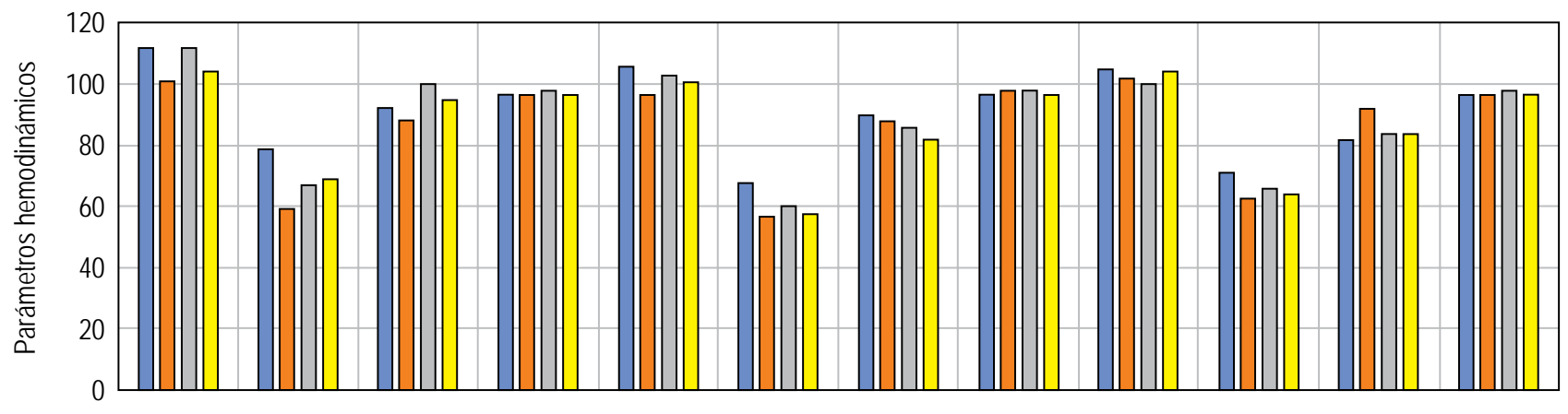

\begin{tabular}{|l|c|c|c|c|c|c|c|c|c|c|c|c|}
\hline & $\begin{array}{c}\text { P. } \\
\text { sistólica }\end{array}$ & $\begin{array}{c}\text { P. } \\
\text { diastólica }\end{array}$ & FC & $\mathrm{O}_{2}$ & $\begin{array}{c}\text { P. } \\
\text { sistólica }\end{array}$ & $\begin{array}{c}\text { P. } \\
\text { diastólica }\end{array}$ & FC & $\mathrm{O}_{2}$ & $\begin{array}{c}\text { P. } \\
\text { sistólica }\end{array}$ & $\begin{array}{c}\text { P. } \\
\text { diastólica }\end{array}$ & FC & $\mathrm{O}_{2}$ \\
\hline$\square$ Grupo I & 112 & 79 & 92 & 97 & 106 & 68 & 90 & 97 & 105 & 71 & 82 & 97 \\
\hline$\square$ Grupo II & 101 & 59 & 88 & 97 & 97 & 57 & 88 & 98 & 102 & 63 & 92 & 97 \\
\hline$\square$ Grupo III & 112 & 67 & 100 & 98 & 103 & 60 & 86 & 98 & 100 & 66 & 84 & 98 \\
\hline$\square$ Grupo IV & 104 & 69 & 95 & 97 & 101 & 58 & 82 & 97 & 104 & 64 & 84 & 97 \\
\hline
\end{tabular}

Figura 1: Parámetros hemodinámicos. P. sistólica = presión arterial sistólica, $\mathrm{mmHg}$; P. diastólica = presión arterial diastólica, $\mathrm{mmHg} ; \mathrm{FC}=$ frecuencia cardíaca Its/min; $\mathrm{O}_{2}=$ oximetría de pulso. 


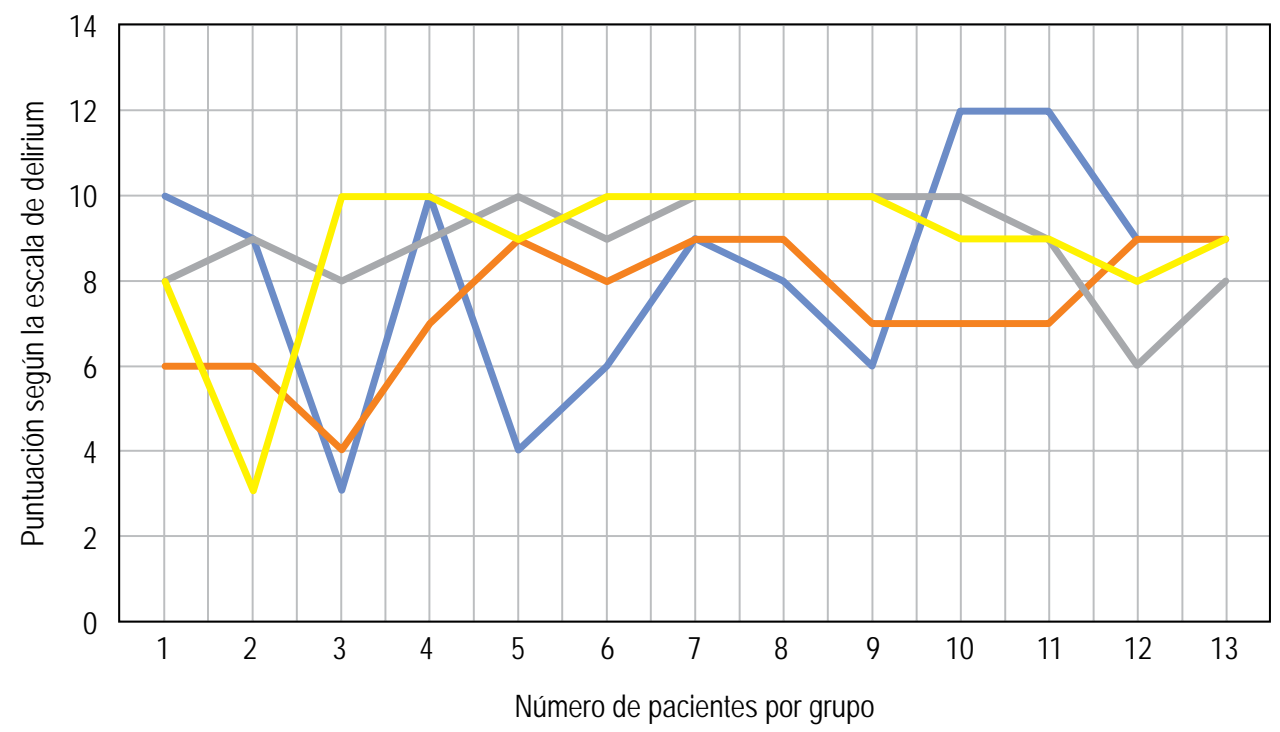

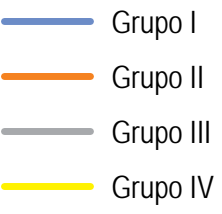

Figura 2:

Escala de delirium del despertar postoperatorio por grupo.

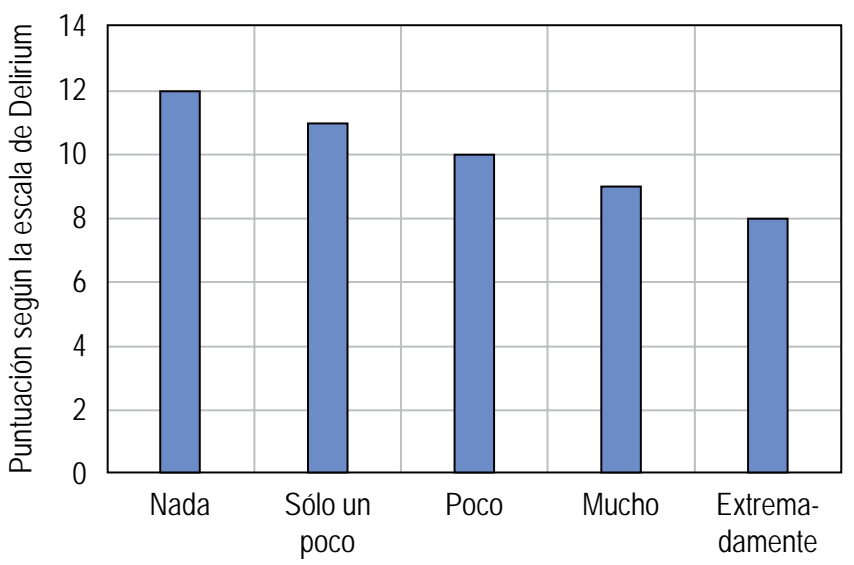

Figura 3: Escala de delirium del despertar postoperatorio.

de canales de calcio, dependientes de voltaje, aumento en el intercambio de sodio hidrogeniones en el interior de las plaquetas y apertura de diferentes tipos de canales de potasio, hiperpolarizando la célula, suprimiendo o disminuyendo la actividad neuronal ${ }^{(5)}$. Su acción nociceptiva está dada por la acción sobre el receptor alfa 2-A adrenérgico de la médula espinal. Su acción hipnótico-sedante a nivel del locus ceruleus mediante su unión a receptores alfa 2-A de este grupo celular ${ }^{(5,6,10)}$.

En los niños, la farmacocinética de la dexmedetomidina es predecible, con una vida media final de 1.8 horas. No se han observado fluctuaciones en frecuencia cardíaca y presión arterial de forma significativa en este grupo de población, ya que esos efectos se relacionan con dosis mayores a las recomendadas o administradas de forma rápida ${ }^{(6)}$.

Estudios recientes sugieren que el efecto analgésico que brinda la dexmedetomidina tiene un componente afectivo-

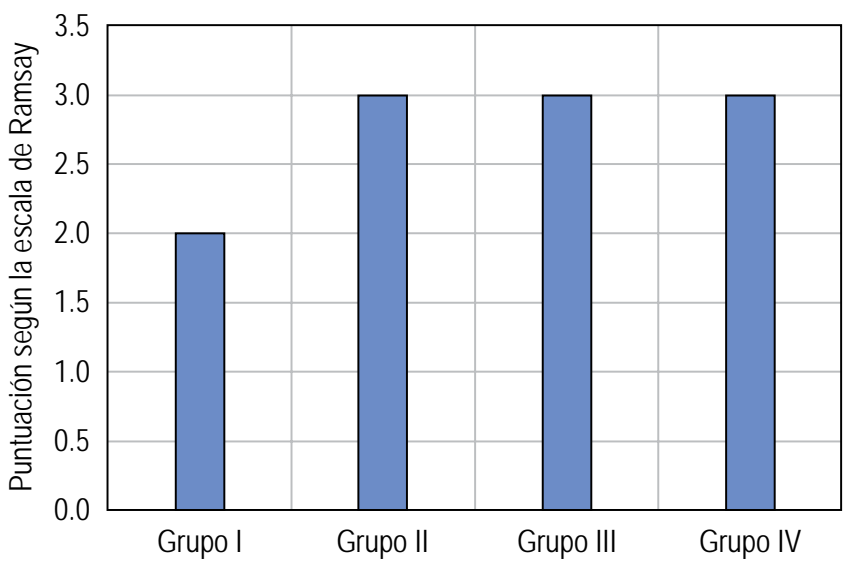

Figura 4: Escala de Ramsay.

emocional del dolor ${ }^{(14)}$. Otros han constatado que al utilizarse como premedicación, reduce los requerimientos de fármacos opioides, hipnóticos y halogenados ${ }^{(5)}$.

La premedicación con dexmedetomidina previa a la cirugía causó una disminución significativa del uso de opioides, y no requirió analgesia suplementaria en el postoperatorio. Se ha demostrado que una dosis administrada después de la técnica anestésica en bolo de $0.5 \mu \mathrm{g} / \mathrm{kg}$ IV reduce el consumo de opioides durante las primeras 24 horas ${ }^{(14,15)}$.

Referente a la comodidad, la cual se entiende como un estado libre de ansiedad o dolor, el paciente pediátrico es más sensible a cambios mínimos en su entorno; la comunicación asertiva con los padres y el niño sobre el procedimiento y los eventos que ocurrirán en el postoperatorio son clave para minimizar la ansiedad y las expectativas que pueden tener sobre el evento anestésico; es por esto que la sedación previa al procedimiento juega un papel importante en el manejo del postoperatorio. 
El delirio postoperatorio tiene una incidencia de 2-80\%, en el cual se reconocen como factores de riesgo para su aparición: masculinos preescolares, emersión anestésica rápida (sevoflurano y desflurano), cirugía de oído, nariz y garganta; y la ansiedad preoperatoria. Se le ha relacionado ampliamente con el dolor y con el grado de ansiedad previa al evento anestésico-quirúrgico, así como con la tasa de aclaramiento, tanto de halogenados como de anestésicos intravenosos y con el emerger. Por ende, el comportamiento del paciente será diferente, según el agente utilizado para su anestesia. Se ha comparado el delirio postoperatorio con los terrores nocturnos ${ }^{(16,17)}$.

En un estudio previo se administró dexmedetomidina en dosis de 0.15 y $0.3 \mu \mathrm{g} / \mathrm{kg}$ IV posterior a la inducción con sevoflurano, y se comparó con un grupo placebo. En ambos grupos, se observó una incidencia reducida en la agitación postoperatoria, sin diferencia en el tiempo de emersión ${ }^{(18)}$.

Se ha utilizado dexmedetomidina en infusión continua de $0.2 \mu \mathrm{g} / \mathrm{kg} / \mathrm{h}$, y se ha visto una disminución en la incidencia de agitación postanestésica con uso de sevoflurano, y de forma similar se ha demostrado su efectividad con dosis única de $0.5 \mu \mathrm{g} / \mathrm{kg}$ previniendo esta complicación ${ }^{(19)}$.

Por lo que podemos concluir que el uso de dexmedetomidina es útil en la medicación preanestésica, ansiólisis, el tratamiento y prevención de dolor, estabilidad hemodinámica, y disminuye los requerimientos de anestésicos y la liberación de catecolaminas; además de prevenir la agitación y el delirio en el postoperatorio en el paciente pediátrico ${ }^{(13,14)}$.

Este es un estudio preliminar, en el que se presenta una serie de casos donde comparamos tres dosis de dexmedetomidina como adyuvante, para valorar la utilidad del fármaco administrado por vía subcutánea sobre sedación, delirio, confort y analgesia del paciente. Al comparar los parámetros hemodinámicos se pudo demostrar que no existe diferencia estadísticamente significativa entre las variables, por lo que la dexmedetomidina por vía subcutánea en las dosis descritas, se puede considerar segura para su administración en el paciente pediátrico, resultados similares a los reportados por Kivisto, $\mathrm{KT}^{(12)}$ y Gutiérrez Vega $\mathrm{A}^{(13)}$ en los que con diferentes objetivos encontraron resultados positivos con la administración de la dexmedetomidina por vía subcutánea.

Referente a la sedación, existen múltiples estudios en los que han sido administradas diferentes dosis de dexme-

Tabla 5: Escala de Confort. Promedio por grupos.

\begin{tabular}{cccc} 
Grupo & Profundo (\%) & Óptimo (\%) & Inadecuado (\%) \\
\hline I & 31 & 69 & 0 \\
II & 85 & 15 & 0 \\
III & 100 & 0 & 0 \\
IV & 100 & 0 & 0
\end{tabular}

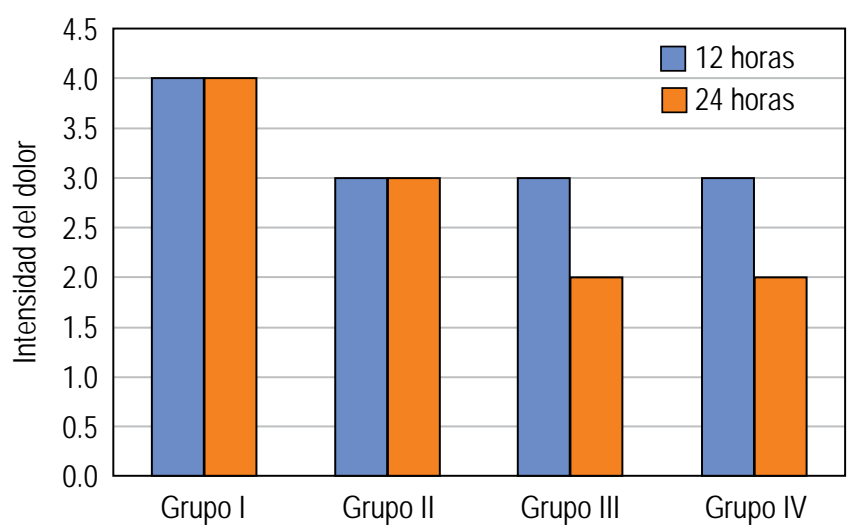

Figura 5: Evaluación de dolor.

detomidina y por diferentes vías con excelentes resultados, obteniendo excelentes grados de sedación, buena ansiólisis y menor delirio, lo que se traduce en mejor comodi$\operatorname{dad}^{(2-4,6,18,19)}$. En nuestro estudio, con la administración subcutánea de dexmedetomidina, los pacientes tuvieron un puntaje en la escala de Ramsay mayor que el grupo placebo, por lo que se puede considerar que la sedación producida por la dexmedetomidina fue independiente de la dosis administrada, obteniendo pacientes tranquilos y cooperadores, siendo la subcutánea una opción de vía de administración a considerar para la sedación del paciente pediátrico.

En relación con la escala de delirium, existen varios informes publicados en los que la administración de dexmedetomidina, tanto como premedicación como administrados en el transoperatorio, disminuyen la aparición de delirio y agitación postanestésica ${ }^{(16-19)}$. En nuestros resultados por vía subcutánea, disminuyó también la incidencia. Llama la atención que con la administración de dexmedetomidina a $1 \mu \mathrm{g} / \mathrm{kg}$, se obtiene mejor puntaje que con dosis mayores, aun en el grupo placebo. Aunque no se pudo demostrar una diferencia estadísticamente significativa.

Respecto a la escala Confort, el mayor grado de comodidad se obtuvo con las dosis mayores de dexmedetomidina (grupo III y IV), $100 \%$ de los pacientes de los grupos se encontró en la categoría de «profundo».

La evaluación del dolor mostró diferencia estadísticamente significativa entre los grupos control (II, III, IV) y el grupo placebo, teniendo mejor analgesia aquéllos que recibieron dexmedetomidina. En la valoración realizada a las 24 horas de la cirugía, se encontró que los pacientes que recibieron la dosis de 1.5 y $2 \mu \mathrm{g} / \mathrm{kg}$ tuvieron mejor analgesia. Los resultados obtenidos con la administración subcutánea mostraron los beneficios de aplicar la dexmedetomidina por esta vía, situación ya demostrada en estudios previos, como el publicado por Schanabel A en 2013, un metaanálisis en el que se encontró que la administración perioperatoria 
mejoraba el control del dolor, y disminuía el consumo de analgésicos al suministrarla por otras vías. Shagufta N, en 2014, reportó disminución de hasta 50\% en el consumo de analgésicos y de $80 \%$ en el requerimiento de dosis de rescate para sedación ${ }^{(14,15)}$.

\section{CONCLUSIONES}

La vida media corta y las diferentes vías de administración de la dexmedetomidina (intravenosa, intranasal, bucal, epidural, subdural, intramuscular, subcutánea) preservan la función respiratoria, ofreciendo seguridad en el paciente pediátrico.
La administración de dexmedetomidina por vía subcutánea en el paciente pediátrico se muestra como una alternativa segura y eficaz para mejorar las condiciones en el postoperatorio, no sólo en el grado de sedación y/o analgesia, sino que en lo general en el grado de comodidad que presentaron los pacientes, esto es un punto importante, mediante el cual puede cambiar la apreciación no sólo de los pacientes, sino también de los padres sobre la experiencia al ser sometidos a una intervención anestésico-quirúrgica.

Queda abierta la posibilidad de realizar más estudios con universos mayores, que den soporte a la vía subcutánea para su uso en el perioperatorio como un medio útil y seguro en la población pediátrica.

\section{REFERENCIAS}

1. López-González BG, Sánchez-Hernández E, Rodríguez-Moreno OA. Dexmedetomidina por vía Subcutánea como coadyuvante anestésico en el paciente pediátrico después de apendicectomía abierta [Tesis]. Guadalajara, México: Universidad de Guadalajara; 2016. No publicada. En archivo de bibliohemeroteca del Hospital Civil de Guadalajara "Fray Antonio Alcalde".

2. Shukry M, Kennedy K. Dexmedetomidine as a total intravenous anesthetic in infants. Paediatr Anaesth. 2007;17:581-583.

3. Munro HM, Tirotta CF, Felix DE, et al. Initial experience with dexmedetomidine for diagnostic and interventional cardiac catheterization in children. Paediatr Anaesth. 2007;17:109-112.

4. Ard J, Doyle W, Bekker A. Awake craniotomy with dexmedetomidine in pediatric patients. J Neurosurg Anesthesiol. 2014;15:263-266.

5. Mato M, Pérez A, Otero J, Torres L. Dexmedetomidina, un fármaco prometedor. Rev Esp Anestesiol Reanim. 2002;49:407-420.

6. Carrillo-Torres O, Pliego-Sánchez MG, Gallegos-Allier MM, et al. Utilidad de la dexmedetomidina en diversos contextos en la medicina actual. Rev Mex Anestesiol. 2014;37:27-34.

7. Cortinez LI, Hsu YW, Sum-Ping ST, Young C, Keifer JC, Macleod D, et al. Dexmedetomidine pharmacodynamics: Part II: Crossover comparison of the analgesic effect of dexmedetomidine and remifentanil in healthy volunteers. Anesthesiology. 2004;101:1077-1083.

8. Snapir A, Posti J, Kentala E, Koskenvuo J, Sundell J, Tuunanen H, et al. Effects of low and high plasma concentrations of dexmedetomidine on myocardial perfusion and cardiac function in healthy male subjects. Anesthesiology. 2006;105:902-910; quiz 1069-1070.

9. Hogue C, Tlake P, Stein P, Richardson C, Domitrovich P, Sessier D. Autonomic nervous system responses during sedative infusions of dexmedetomidina. Anesthesiology. 2002;97:592-598.
10. Ramsay M. Bariatric surgery: The role of dexmedetomidine. Critical Care Medicine. 2006;25:51-56.

11. Brunton LL, Lazo JS, Parker KL. Goodman \& Gilman. Las bases farmacológicas de la terapéutica. 11 edición. Mgraw-Hill; 2010. pp. 4-6.

12. Kivisto KT, Kallio A, Neuvonen PJ. Pharmacokinetics and pharmacodynamics of transdermal dexmedetomidine Eur J Clin Pbarmacol. 1994;46:345-349.

13. Gutiérrez-Vega A, Grandeño-Vega A, Villareal-Careaga J. Dexmedetomidina subcutánea para disminuir los requerimientos de halogenados y narcóticos en anestesia general balanceada, para cirugía de mastectomía. Arch Salud Sin. 2011;5:13-18.

14. Schanabel A, Reichi SU, Poepping DM, et al. Efficacy and safety of intraoperative dexmedetomidne for acute postoperative pain in children. Paediatr Anaesth. 2013;23:170-179.

15. Shagufta N, Erum O. Dexmedetomidine in current anaestesia practice: a review. J Clin Diagn Res. 2014;8:GE01-GE04.

16. Martin JC, Liley DT, Davidson AJ, Sanders RD, Sleigh JW. Multichannel brain electrical activity in a 5 year old preceding an episode of emergence delirium. ASA Annual congress abstract A951, 2012.

17. Dahmani S, Delivet H, Hilly J. Emergence delirium in children: an update. Curr Opin Anaesthesiol. 2014;27:309-315.

18. Ibacache ME, Munoz HR, Brandes V, Morales AL. Single-dose dexmedetomidine reduces agitation after sevoflurane anesthesia in children. Anesth Analg. 2004;98:60-63.

19. Guler G, Akin A, Tosun Z, Ors S, Esmaoglu A, Boyaci A. Single-dose dexmedetomidine reduces agitation and provides smooth extubation after pediatric adenotonsillectomy. Paediatr Anaesth. 2005;15:762766. 